\title{
Prognostic significance of systemic immune-inflammation index- based nomogram for early stage hepatocellular carcinoma after radiofrequency ablation
}

\author{
Yujing Xin ${ }^{1}$, Yi Yang ${ }^{1}$, Ning Liu ${ }^{2}$, Yi Chen ${ }^{1,3}$, Yanan Wang ${ }^{1}$, Xinyuan Zhang ${ }^{1}$, Xiao Li $^{1}$, Xiang Zhou ${ }^{1}$ \\ ${ }^{1}$ Department of Interventional Therapy, National Cancer Center/National Clinical Research Center for Cancer/Cancer Hospital, Chinese Academy \\ of Medical Sciences and Peking Union Medical College, Beijing, China; ${ }^{2}$ Department of Computer Science and Technology, Tsinghua University, \\ Beijing, China; ${ }^{3}$ Department of Interventional Radiology, First Hospital of Shanxi Medical University, Taiyuan, China \\ Contributions: (I) Conception and design: Y Xin; (II) Administrative support: Y Xin, Y Yang, N Liu; (III) Provision of study materials or patients: Y \\ Xin, Y Yang; (IV) Collection and assembly of data: Y Xin, Y Chen; (V) Data analysis and interpretation: Y Xin, N Liu; (VI) Manuscript writing: All \\ authors; (VII) Final approval of manuscript: All authors. \\ Correspondence to: Xiang Zhou, MD. Department of Interventional Therapy, National Cancer Center/National Clinical Research Center for Cancer/ \\ Cancer Hospital, Chinese Academy of Medical Sciences and Peking Union Medical College, Beijing 100021, China. Email: xiangzhousmart@163.com.
}

Background: Radiofrequency ablation (RFA) is the recommended treatment for early stage hepatocellular carcinoma (HCC), and the prognostic value of systemic immune-inflammation index (SII) in early stage HCC is not discussed. Therefore, the purpose of the study is to explore the prognostic value of SII based on lymphocyte, neutrophil, and platelet counts in patients with HCC after RFA.

Methods: We retrospectively evaluated the prognostic value of the SII in training and validation cohorts, and then established an effective nomogram for HCC after RFA based on SII. The C-index, and area under the time-dependent receiver operating characteristic curve (t-AUC) were used to evaluate the discrimination and calibration value of the nomogram.

Results: An optimal cut-off value for the SII of $324.55 \times 10^{9}$ stratified the patients with HCC into highand low-SII groups. Univariate and multivariate analyses revealed that SII was an independent predictor for overall survival (OS) and recurrence-free survival (RFS). Moreover, SII was an independent prognostic factor for early-stage HCC with normal alpha-fetoprotein (AFP) levels. The t-AUC of the SII was higher for OS and RFS than for neutrophil-lymphocyte ratio (NLR) and platelet-lymphocyte ratio (PLR). A high preoperative SII was associated with multiple tumors, larger tumors, and higher levels of AFP. A welldiscriminated and calibrated nomogram was constructed to predict the probability of 1-, 2-, 3-, and 5-year RFS with C-indexes of 0.80 , which was significantly higher than that obtained with other prognostic clinical indexes.

Conclusions: The SII is an independent prognostic factor affecting the survival outcomes of patients with early-stage HCC. The comprehensive nomogram based on SII presented in this study is a promising model for predicting RFS in HCC patients after RFA.

Keywords: Hepatocellular carcinoma (HCC); radiofrequency ablation (RFA); systemic immune-inflammation index (SII); recurrence-free survival (RFS); prognostic nomogram

Submitted Aug 20, 2020. Accepted for publication Nov 27, 2020.

doi: 10.21037/jgo-20-342

View this article at: http://dx.doi.org/10.21037/jgo-20-342 


\section{Introduction}

Hepatocellular carcinoma (HCC) accounts for 75-85\% of all primary liver malignancies $(1,2)$. It is the sixth most common type of cancer and the fourth most common cause of cancer-related death worldwide (3). China accounts for more than $50 \%$ of the global burden of HCC, with an estimated incidence of 369,000 new cases and 366,000 deaths each year (1-4). At present, surgical resection, ablation, and liver transplantation are recommended as the first-line radical treatments for HCC that meets the Milan criteria (5-10). However, the long-term prognosis of HCC patients is still unsatisfactory due to the high incidence rate of tumor recurrence or distant metastasis: up to $70 \%$ within 5 years after radical therapy $(8,11)$.

Radiofrequency ablation (RFA) is the recommended treatment for early stage HCC, especially in patients who are not suitable for liver resection or liver transplantation (7). However, studies have found higher recurrence rate and poorer long-term survival in patients with HCC after RFA, compared to patients with HCC after surgical resection (12-15). Therefore, accurate prediction of the risk of tumor recurrence after RFA for early-stage HCC and timely intervention are particularly important for improving the overall survival (OS) of HCC patients.

Many studies have found that preoperative inflammatory marker-based scoring systems, such as the Glasgow prognostic score (GPS), platelet lymphocyte ratio (PLR), prognostic nutritional index (PNI), and neutrophil lymphocyte ratio (NLR), can be used to predict the risk of tumor recurrence in cancer patients $(16,17)$. Tumor-related inflammatory and immune responses are considered as vital for tumor occurrence, development, angiogenesis, and metastasis $(18,19)$. A novel systemic immune inflammatory index (SII) based on absolute lymphocyte, platelet, and neutrophil counts in peripheral blood has been reported to be an ideal prognostic indicator for multiple cancers, including HCC (20-22). Many studies have demonstrated that elevated SII values are associated with poor outcomes in HCC patients undergoing surgical resection and liver transplantation $(23,24)$. However, the threshold for SII values ranges from 200 to 600 across the studies (25-27). The prognostic value of SII in patients with early-stage HCC is still unclear.

This retrospective study was conducted to investigate the prognostic value of pre-procedure SII score in patients with HCC meeting the Milan criteria after RFA treatment. A novel comprehensive nomogram based on SII score was developed to predict the probability of RFS in these patients to help clinical oncologists in formulating adjuvant therapeutic and preventive strategies. We present the following article in accordance with the STROBE reporting checklist (available at: http://dx.doi. org/10.21037/jgo-20-342).

\section{Methods}

The study was conducted in accordance with the Declaration of Helsinki (as revised in 2013). The study was approved by the Institutional Review Boards of the National Cancer Center (NCC2019KZ-010). All patients included in this study signed their informed consent to treatment. The need for written informed consent to publish the data was waived by the Ethics Committees, since the personal details of the patients were kept confidential.

\section{Patients}

Patients who refused to undergo surgery and those who were unfit for surgery received RFA treatment after diagnosis with HCC. The inclusion criteria were as follows: (I) HCC confirmed by histology or the noninvasive diagnostic criteria recommended by the European Association for the Study of Liver (EASL) (28); (II) Eastern Cooperative Oncology Group (ECOG) performance status of 0 or 1; (III) solitary tumor with diameter $\leq 5 \mathrm{~cm}$ or $\leq 3$ tumors, each with diameter $\leq 3 \mathrm{~cm}$ (Milan criteria); (IV) absence of extrahepatic metastasis and major vessel invasion; (V) complete tumor ablation with RFA. The exclusion criteria were: (I) loss to follow-up; (II) tumors that did not meet the Milan criteria; (III) patients who received surgery as the primary treatment for HCC; and (IV) first RFA performed at other centers.

\section{Data collection}

Neutrophil, lymphocyte, and platelet counts and other clinically relevant variables were measured from the peripheral blood $\leq 1$ week before RFA. The variables measured included maximum tumor size, number of tumors, presence of cirrhosis, hepatitis B virus (HBV) infection, Child-Turcotte-Pugh (CTP) grade, Model for End-Stage Liver Disease (MELD) score, serum alpha-fetoprotein (AFP), total bilirubin (TBIL), aspartic transaminase (AST), alanine aminotransferase (ALT), and serum $\gamma$-glutamyl transpeptidase $(\gamma-G T)$ levels, albumin (ALB). 
In this study, four scoring systems (SII, PNI, PLR, and NLR) were evaluated in terms of their ability to predict the prognosis of patients with HCC after RFA. The formulas used to calculate these scores were as follows:

(I) $\quad$ PNI $=10 \times$ albumin $(\mathrm{g} / \mathrm{dL})+0.005 \times$ total lymphocyte count $(/ \mu \mathrm{L})$;

(II) $\mathrm{NLR}=$ neutrophil count/lymphocyte count;

(III) SII = platelet count $\times$ neutrophil count/lymphocyte count;

(IV) PLR = platelet count/lymphocyte count.

\section{RFA procedure}

All RFA procedures were performed under ultrasound or computed tomography (CT) guidance by doctors with at least 5 years of RFA experience using a radiofrequency generator system (S-1500, MedSphere International Inc., CA, USA). Local anesthesia was given before RFA. The type of electrode to be used (monopolar and/or umbrella) (MedSphere International Inc, CA, USA), the power of the generator, and ablation strategy were determined by the operator after consideration of tumor burden and the manufacturer's recommendations. Expanding or overlapping ablation was performed such that all tumors were ablated with a safety margin of at least $0.5 \mathrm{~cm}(29,30)$. Artificial ascites was created before RFA to provide an insulation layer between the tumor(s) and the vital organs if the tumor(s) were located in unfavorable locations. In order to ensure complete ablation, postoperative contrast-enhanced (CE) imaging, such as magnetic resonance imaging (MRI), CT, or ultrasound were performed immediately after RFA. If residual tumor was present, additional RFA was performed to achieve complete ablation. After tumor ablation, the ablation path was cauterized to avoid tumor seeding and hemorrhage during the RFA procedure.

\section{Follow-up}

Patients were followed up every three months in the first year after RFA, and every six months in subsequent years until tumor recurrence or death. The last follow-up date for the present study was December 30, 2019. Each follow-up visit consisted of a clinical interview, physical examination, routine blood investigations, liver function tests, serum AFP, and CECT (chest and abdomen). If distant metastasis was suspected on CT imaging, whole-body positron emission tomography-computed tomography (PET-CT) and/or bone scan was performed to obtain a definitive diagnosis. For patients with chronic (HBV) infection [positive hepatitis B surface antigen (HBsAg) and HBV DNA level higher than 1,000 copies $/ \mathrm{mL}$ ], daily oral antiviral therapy with entecavir, adefovir dipivoxil, or lamivudine was prescribed (31). For patients with chronic hepatitis $\mathrm{C}$ virus (HCV) infection (positive serum HCV RNA), combination treatment with ribavirin and interferon was prescribed (32).

The primary outcomes included recurrence-free survival (RFS) and OS. RFS was defined as the time interval between RFA treatment and first tumor recurrence. OS was defined as the duration from the first ablation therapy to death from any cause or last follow-up. A multidisciplinary approach, which included surgical resection, systemic therapy (i.e., sorafenib), repeat RFA, or transarterial chemoembolization (TACE), was adopted for the treatment of recurrence (5).

\section{Statistical analysis}

Continuous variables were compared using the MannWhitney U-test or the $t$-test. Categorical variables were analyzed either by $\chi^{2}$ test or Fisher's exact test. The KaplanMeier curve was used to evaluate the cumulative incidence rates of RFS and OS, and the log-rank test was used to evaluate differences between groups. The risk factors for OS and RFS were obtained with univariate and multivariate Cox proportional hazards models. A multivariable Cox hazard model was constructed by using the significant variables $(\mathrm{P}<0.1)$ from the univariable Cox regression. The optimal cut-off value for SII, PLR, and NLR was determined with $\mathrm{X}$-tile 3.6.1 software (https://medicine. yale.edu/lab/rimm/research/software/) (33). A two-tailed 95\% confidence interval (CI) was applied to reveal the accuracy of the hazard ratio (HR), and the statistically significant level was set as 0.05. GraphPad Prism 7.0 software (La Jolla, CA, USA) and R software (version 3.6.2) were used to perform the statistical analyses.

The nomogram was plotted by selecting significant variables with $\mathrm{P}<0.05$ on multivariate Cox proportional hazards model analysis. Five hundred bootstrap resamples were performed on the named graph for internal validation of the database. The concordance index (C-index) was computed to evaluate the performance of these models in predicting prognosis. The $\mathrm{C}$-index ranges from 0.5 to 1.0 , with 0.5 suggesting the model performs like a random choice and 1.0 suggesting the model is able to correctly predict outcomes. The nomogram for 1-, 2-, 3-, and 5-year RFS was calibrated by comparing observed survival with predicted survival. The predictive power of the model was 
Table 1 Baseline characteristics of HCC patients in the training and validation cohorts

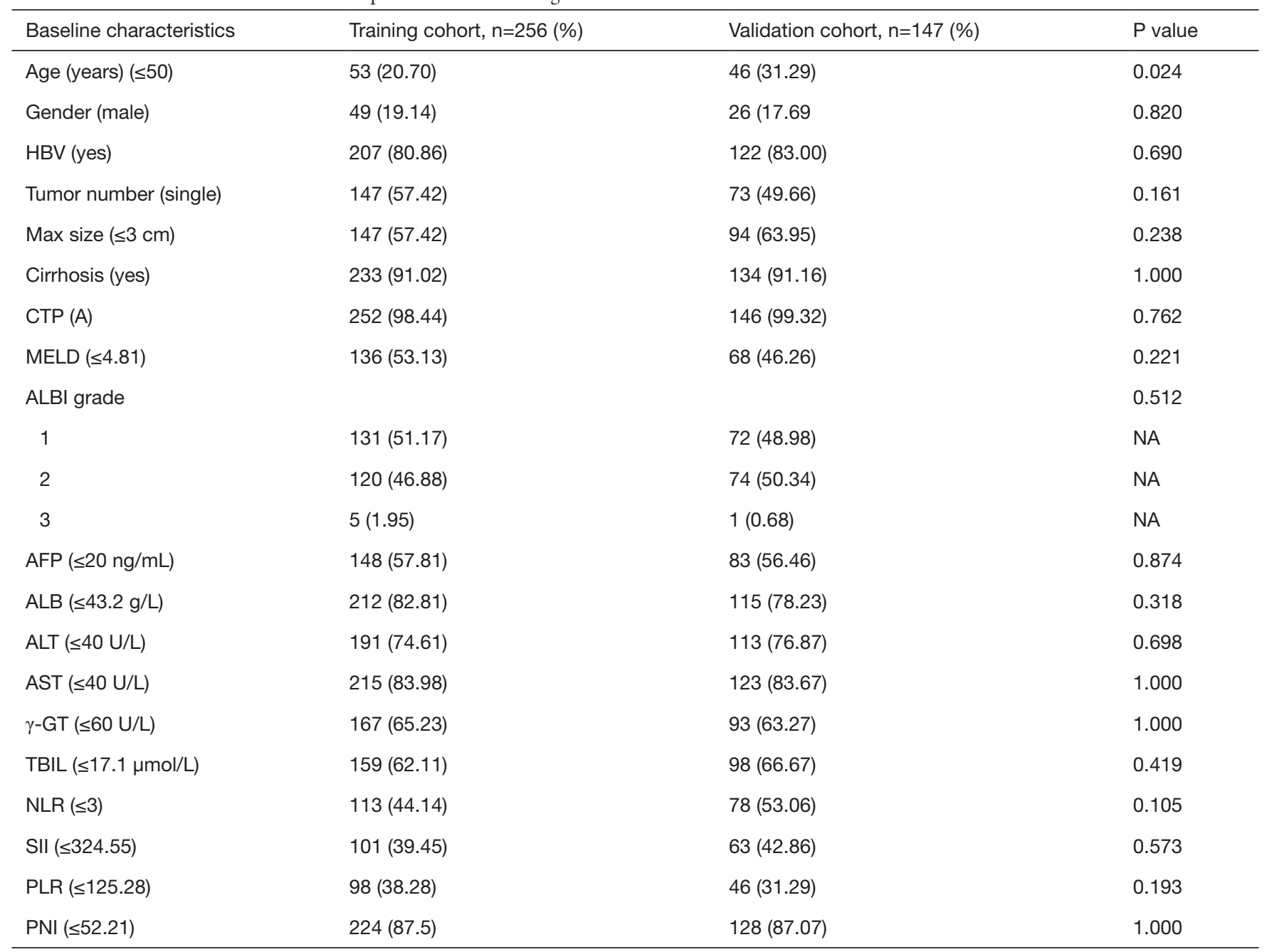

HCC, hepatocellular carcinoma; HBV, hepatitis B virus; CTP, Child-Turcotte-Pugh; MELD, Model for End-Stage Liver Disease; AFP, alphafetoprotein; ALB, albumin; ALT, alanine aminotransferase; AST, aspartic transaminase; $\gamma$-GT, $\gamma$-glutamyl transpeptidase; TBIL, total bilirubin; NLR, neutrophil lymphocyte ratio; SII, systemic immune inflammatory index; PLR, platelet lymphocyte ratio; PNI, prognostic nutritional index.

analyzed by examination of the time-dependent receiver operating characteristic (t-ROC) curve. The $\mathrm{C}$ index and area under the t-ROC (t-AUC) were computed to quantitatively compare methods.

\section{Results}

\section{Patient demographics}

A total of 509 early-stage HCC patients received RFA at two Chinese hospitals during the study period (from January 2014 to December 2019). Among these patients,
106 were excluded because the HCC was beyond Milan criteria $(n=33)$, the ablation was incomplete $(n=38)$, or the patient was lost to follow up ( $\mathrm{n}=35$ ). Finally, 256 patients with HCC who had undergone RFA at the National Cancer Center were selected as the training cohort. Another 147 patients with HCC from First Hospital of Shanxi Medical University were enrolled as the validation cohort. The demographics of the study patients are shown in Table 1 . The baseline clinical characteristics of the training and validation cohorts were similar, except for age (Table 1).

In the training cohort, the optimal cut-off value for 
Table 2 Univariate and multivariate Cox regression analyses for the training cohort

\begin{tabular}{|c|c|c|c|c|c|c|c|c|}
\hline \multirow{2}{*}{ Variable } & \multicolumn{4}{|c|}{ Univariate analysis } & \multicolumn{4}{|c|}{ Multivariate analysis } \\
\hline & $\mathrm{HR}(95 \% \mathrm{Cl})$ & $\mathrm{P}$ & $\mathrm{HR}(95 \% \mathrm{Cl})$ & $\mathrm{P}$ & $\mathrm{HR}(95 \% \mathrm{Cl})$ & $\mathrm{P}$ & $\mathrm{HR}(95 \% \mathrm{Cl})$ & $\mathrm{P}$ \\
\hline $\begin{array}{l}\text { Age, y ( } \leq 50 \text { vs. } \\
>50)\end{array}$ & $0.92(0.590-1.440)$ & 0.720 & $1.418(0.928-2.166)$ & 0.107 & NA & NA & NA & NA \\
\hline $\begin{array}{l}\text { Gender (male } \\
\text { vs. female) }\end{array}$ & $1.286(0.775-2.132)$ & 0.330 & $1.160(0.724-1.859)$ & 0.538 & NA & NA & NA & NA \\
\hline HBV & $0.710(0.447-1.129)$ & 0.148 & $0.761(0.486-1.192)$ & 0.233 & NA & NA & NA & NA \\
\hline $\begin{array}{l}\text { AFP, } n g / m L \\
(\leq 20 \text { vs. }>20)\end{array}$ & $3.811(2.565-5.664)$ & $<0.001$ & $2.786(1.911-4.061)$ & $<0.001$ & $2.245(1.447-3.482)$ & $<0.001$ & $1.813(1.177-2.790)$ & $<0.001$ \\
\hline $\begin{array}{l}\text { Tumor number } \\
\text { (single vs. } \\
\text { multiple) }\end{array}$ & $1.964(1.346-2.866)$ & $<0.001$ & $1.617(1.131-2.310)$ & $<0.001$ & $1.709(1.127-2.590)$ & 0.012 & $1.367(0.929-2.011)$ & 0.113 \\
\hline Cirrhosis & $0.9293(0.469-1.841)$ & 0.834 & $0.737(0.384-1.411)$ & 0.357 & NA & NA & NA & NA \\
\hline CTP (A vs. B) & $0.7377(0.103-5.295)$ & 0.762 & $1.996(0.274-14.530)$ & 0.495 & NA & NA & NA & NA \\
\hline $\begin{array}{l}\text { MELD }(\leq 4.81 \\
\text { vs. }>4.81)\end{array}$ & $0.693(0.473-1.015)$ & 0.059 & $0.797(0.555-1.143)$ & 0.217 & NA & NA & NA & NA \\
\hline ALBI & & 0.8 & & 0.2 & NA & NA & NA & NA \\
\hline 2 & $1.132(0.774-1.656)$ & 0.523 & $1.358(0.945-1.951)$ & 0.098 & NA & NA & NA & NA \\
\hline 3 & $0.882(0.215-3.621)$ & 0.862 & $2.355(0.566-9.791)$ & 0.239 & NA & NA & NA & NA \\
\hline $\begin{array}{l}\mathrm{ALT}, \mathrm{U} / \mathrm{L}(\leq 40 \\
\text { vs. }>40)\end{array}$ & $1.209(0.794-1.842)$ & 0.377 & $1.142(0.765-1.705)$ & 0.515 & NA & NA & NA & NA \\
\hline $\begin{array}{l}\text { ALB, g/L } \\
(\leq 43.2 \text { vs. } \\
>43.2)\end{array}$ & $1.399(0.895-2.186)$ & 0.141 & $0.9748(0.627-1.516)$ & 0.910 & NA & NA & NA & NA \\
\hline $\begin{array}{l}\text { PLR }(\leq 125.28 \\
\text { vs. }>125.28)\end{array}$ & 2.132 (1.393-3.262) & $<0.001$ & $1.656(1.118-2.454)$ & 0.012 & $0.736(0.422-1.282)$ & 0.279 & $0.738(0.450-1.212)$ & 0.230 \\
\hline $\begin{array}{l}\text { NLR ( } \leq 3 \text { vs. } \\
>3)\end{array}$ & $2.250(1.497-3.382)$ & $<0.001$ & $1.884(1.441-3.706)$ & $<0.001$ & $1.433(0.877-2.342)$ & 0.151 & $1.166(0.750-1.814)$ & 0.495 \\
\hline $\begin{array}{l}\mathrm{PNI}(\leq 52.21 \text { vs. } \\
>52.21)\end{array}$ & $0.593(0.318-1.107)$ & 0.101 & $0.568(0.313-1.032)$ & 0.063 & $1.109(0.556-2.213)$ & 0.770 & $0.816(0.422-1.578)$ & 0.546 \\
\hline $\begin{array}{l}\text { SII }(\leq 324.55 \\
\text { vs. }>324.55)\end{array}$ & 5.717 (3.391-9.640) & $<0.001$ & $3.676(2.275-5.938)$ & $<0.001$ & $4.072(2.271-7.304)$ & $<0.001$ & $2.619(1.501-4.571)$ & $<0.001$ \\
\hline
\end{tabular}

OS, overall survival; RFS, recurrence-free survival; HR, hazard ratio; Cl, confidence interval; HBV, hepatitis B virus; AFP, alphafetoprotein; CTP, Child-Turcotte-Pugh; MELD, Model for End-Stage Liver Disease; ALB, albumin; ALT, alanine aminotransferase; AST, aspartic transaminase; $\gamma$-GT, $\gamma$-glutamyl transpeptidase; TBIL, total bilirubin; NLR, neutrophil lymphocyte ratio; SII, systemic immune inflammatory index; PLR, platelet lymphocyte ratio; PNI, prognostic nutritional index. 

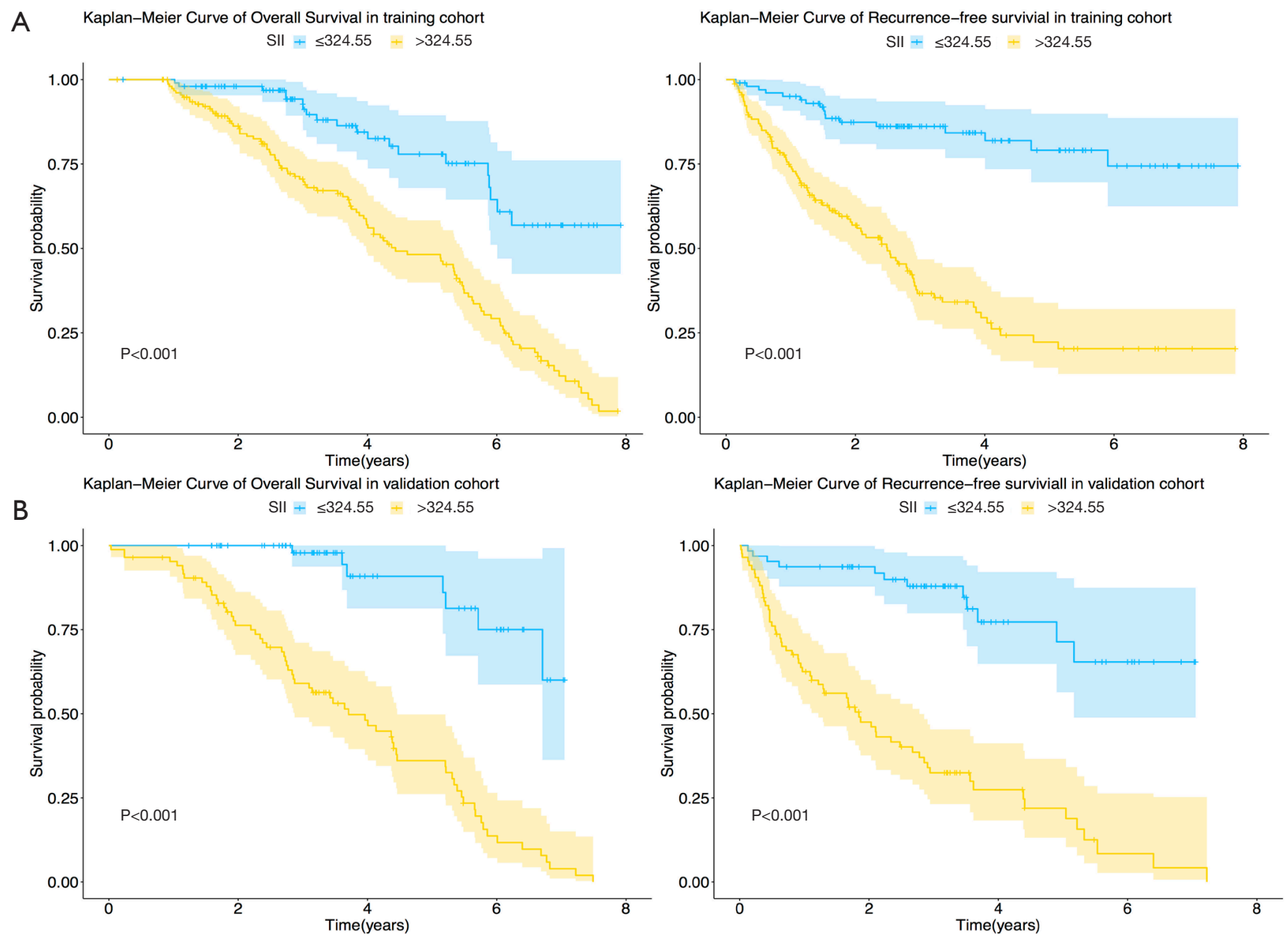

Figure 1 Prognostic significance of SII in HCC patients after RFA. The results of Kaplan-Meier analysis of OS and RFS based on the SII in the training (A) and validation (B) cohorts. SII, systemic immune-inflammation index; HCC, hepatocellular carcinoma; RFA, radiofrequency ablation; OS, overall survival; RFS, recurrence-free survival.

SII was $324.55 \times 10^{9}$. In the training group, $101(39.45 \%)$ patients had high SII scores $\left(>324 \times 10^{9}\right)$. In the validation group, the number of patients with high SII scores was 63 $(42.86 \%)$.

In the training cohort, the 1-, 3- and 5-year OS were $98.4 \%, 78.7 \%$, and $59.0 \%$, respectively, with median followup duration of 43.9 months (range, 12.6-95.0 months). During the follow-up period, recurrence developed in 109 HCC patients.

In the validation cohort, the 1-year, 3-year, and 5-year OS were $97.3 \%, 75.1 \%$, and $55.5 \%$, respectively. The median follow-up duration was 41.7 months (range, 14.884.6 months). During the follow-up period, recurrence developed in 74 HCC patients.

\section{Prognostic value of SII in the training cohort}

The results of univariate Cox proportional hazard model analysis demonstrated that AFP level, tumor size, tumor number, $\gamma$-GT, PLR, NLR, and SII were associated with OS and RFS in the training cohort (Table 2). A low level of preoperative SII was related to both prolonged RFS (HR, 5.717; 95\% CI, 3.391-9.640; $\mathrm{P}<0.001)$ and OS (HR, 3.676; 95\% CI, 2.275-5.938; P<0.001). The Kaplan-Meier curves illustrated that elevated SII scores were associated with shorter RFS $(\mathrm{P}<0.001$, Figure 1) and $\mathrm{OS}(\mathrm{P}<0.001)$. The 
Table 3 The time-dependent AUC of inflammatory indexes in the training and validation cohorts

\begin{tabular}{|c|c|c|c|c|c|}
\hline Variable & 12 months & 24 months & 36 months & 60 months & C-index \\
\hline \multicolumn{6}{|c|}{ Training cohort } \\
\hline SII & $0.677(0.617-0.734)$ & $0.683(0.622-0.742)$ & $0.742(0.680-0.804)$ & $0.777(0.695-0.858)$ & $0.694(0.633-0.754)$ \\
\hline PLR & $0.633(0.569-0.697)$ & $0.6021(0.537-0.668)$ & $0.605(0.535-0.674)$ & $0.608(0.515-0.700)$ & $0.607(0.542-0.672)$ \\
\hline NLR & $0.638(0.569-0.707)$ & $0.600(0.532-0.668)$ & $0.613(0.541-0.684)$ & $0.677(0.589-0.766)$ & $0.624(0.558-0.691)$ \\
\hline CTP & $0.496(0.472-0.520)$ & $0.497(0.483-0.512)$ & $0.501(0.487-0.516)$ & $0.496(0.489-0.504)$ & $0.498(0.484-0.512)$ \\
\hline \multicolumn{6}{|c|}{ Validation cohort } \\
\hline SII & $0.712(0.641-0.782)$ & $0.763(0.696-0.829)$ & $0.752(0.676-0.829)$ & $0.713(0.590-0.836)$ & $0.758(0.687-0.828)$ \\
\hline PLR & $0.610(0.536-0.683)$ & $0.608(0.535-0.682)$ & $0.602(0.525-0.680)$ & $0.634(0.509-0.7600)$ & $0.628(0.557-0.700)$ \\
\hline CTP & $0.486(0.458-0.512)$ & $0.489(0.469-0.510)$ & $0.492(0.477-0.508)$ & $0.494(0.482-0.506)$ & $0.491(0.473-0.509)$ \\
\hline
\end{tabular}

NLR, neutrophil lymphocyte ratio; SII, systemic immune inflammatory index; PLR, platelet lymphocyte ratio; CTP, Child-Turcotte-Pugh; MELD, Model for End-Stage Liver Disease.

median OS of patients in the high SII and low SII groups were 38.8 and 44.0 months, respectively. The median RFS of patients in the high SII and low SII groups was 19.7 and 36.3 months, respectively.

Multivariate analysis indicated that the SII was an independent predictor for RFS (HR, 4.072; 95\% CI, 2.271-7.304; $\mathrm{P}<0.001)$ and $\mathrm{OS}(\mathrm{HR}, 2.619$; 95\% CI, 1.5014.571; $\mathrm{P}<0.001$; Table 2). Among the three indexes based on preoperative levels of inflammation and two traditional liver function reserve models, the SII achieved the highest C-index for predicting RFS in HCC patients (Table 3).

\section{Validation of the prognostic value of SII in an independent set}

The prognostic significance of preoperative SII was further verified in an independent validation cohort of 147 patients. The results obtained in the validation cohort were similar to those obtained in the training cohort. Univariate and multivariate Cox proportional hazard models showed that SII was an independent prognostic factor associated with RFS and OS in HCC patients (Table 4). The highest C-index was seen with SII for the prediction of 1-, 2-, 3-, and 5-year RFS in early-stage HCC patients after ablation (Table 4).

\section{Relationship between SII and clinicopathological features}

The relationship between SII and clinical baseline characteristics is shown in Table 5. Briefly, elevated preoperative SII was associated with larger tumor size, multiple tumors, higher levels of AFP, and high recurrence rate in the training and validation cohorts.

\section{Prognostic value of the SII in early-stage HCC patients with normal AFP levels}

We performed subgroup analysis to explore the relationship between SII and survival in patients with early-stage HCC with normal AFP levels. In the training cohort, patients with normal AFP levels and low preoperative SII had longer RFS and OS compared to those with high SII (Figure $2 A, \mathrm{P}<0.001$ and $\mathrm{P}<0.001$, respectively). Similarly, in the validation cohort, high SII levels indicated poorer RFS and OS in HCC patients with normal AFP levels (Figure 2B, $\mathrm{P}<0.001$ and $\mathrm{P}=0.002$, respectively).

\section{Establishment and verification of the nomogram}

A comprehensive nomogram to predict 1-, 2-, 3-, and 5 -year RFS rates was constructed based on multivariate Cox regression analysis to include tumor number, tumor size, AFP, and SII score (Figure 3). The calibration curves for predicting 1-, 2-, 3-and 5-year RFS after RFA in the training and validation sets are shown in Figure $4 A$.

In the training cohort, the C-index for the likelihood of 
Table 4 Univariate and multivariate Cox regression analyses in the validation cohort

\begin{tabular}{|c|c|c|c|c|c|c|c|c|}
\hline \multirow{2}{*}{ Variable } & \multicolumn{4}{|c|}{ Univariate analysis } & \multicolumn{4}{|c|}{ Multivariate analysis } \\
\hline & $\mathrm{HR}(95 \% \mathrm{Cl})$ & $\mathrm{P}$ & $\mathrm{HR}(95 \% \mathrm{Cl})$ & $\mathrm{P}$ & $\mathrm{HR}(95 \% \mathrm{Cl})$ & $\mathrm{P}$ & $\mathrm{HR}(95 \% \mathrm{Cl})$ & $\mathrm{P}$ \\
\hline Age, y ( $\leq 50$ vs. $>50)$ & $1.147(0.697-1.890)$ & 0.589 & $1.492(0.894-2.490)$ & 0.126 & NA & NA & NA & NA \\
\hline $\begin{array}{l}\text { Gender (male vs. } \\
\text { female) }\end{array}$ & $1.136(0.579-2.229)$ & 0.712 & $0.913(0.477-1.758)$ & 0.783 & NA & NA & NA & NA \\
\hline $\begin{array}{l}\text { AFP, ng/mL ( } \leq 20 \text { vs. } \\
>20)\end{array}$ & 4.309 (2.602-7.133) & $<0.001$ & $5.157(2.946-9.028)$ & $<0.001$ & $\begin{array}{c}2.017(1.049- \\
3.880)\end{array}$ & 0.035 & $\begin{array}{c}2.208(1.093- \\
4.458)\end{array}$ & 0.027 \\
\hline $\begin{array}{l}\text { Tumor number } \\
\text { (single vs. multiple) }\end{array}$ & $2.496(1.530-4.072)$ & $<0.001$ & $2.137(1.296-3.524)$ & 0.003 & $\begin{array}{c}1.748(0.988- \\
3.093)\end{array}$ & 0.055 & $\begin{array}{c}1.479(0.810- \\
2.703)\end{array}$ & 0.203 \\
\hline CTP (A vs. B) & $4.671(0.635-34.330)$ & 0.13 & $23.63(2.845-196.300)$ & 0.003 & NA & NA & NA & NA \\
\hline $\begin{array}{l}\text { MELD ( } \leq 4.81 \text { vs. } \\
4.81)\end{array}$ & $1.309(0.821-2.088)$ & 0.258 & $1.006(0.627-1.613)$ & 0.981 & NA & NA & NA & NA \\
\hline ALBI & & 0.2 & & 0.6 & NA & NA & NA & NA \\
\hline 2 & $1.099(0.691-1.746)$ & 0.691 & $1.251(0.782-2.003)$ & 0.350 & NA & NA & NA & NA \\
\hline 3 & $7.103(0.940-53.666)$ & 0.0574 & $1.932(0.261-14.273)$ & 0.519 & NA & NA & NA & NA \\
\hline $\begin{array}{l}\mathrm{ALT}, \mathrm{U} / \mathrm{L}(\leq 40 \text { vs. } \\
>40)\end{array}$ & $0.923(0.534-1.594)$ & 0.773 & $0.637(0.361-1.124)$ & 0.120 & NA & NA & NA & NA \\
\hline $\begin{array}{l}\text { AST, U/L ( } \leq 40 \text { vs. } \\
>40)\end{array}$ & $1.235(0.677-2.252)$ & 0.491 & $1.053(0.576-1.927)$ & 0.866 & NA & NA & NA & NA \\
\hline $\begin{array}{l}\operatorname{PLR}(\leq 125.28 \text { vs. } \\
>125.28)\end{array}$ & $2.379(1.327-4.267)$ & 0.004 & $1.873(1.042-3.370)$ & 0.036 & $\begin{array}{c}1.285(0.588- \\
2.809)\end{array}$ & 0.530 & $\begin{array}{c}1.078(0.492- \\
2.363)\end{array}$ & 0.851 \\
\hline $\operatorname{NLR}(\leq 3$ vs. $>3)$ & $1.404(0.876-2.250)$ & 0.158 & $1.378(0.852-2.228)$ & 0.192 & $\begin{array}{c}1.378(0.794- \\
2.389)\end{array}$ & 0.254 & $\begin{array}{c}1.508(0.870- \\
2.613)\end{array}$ & 0.143 \\
\hline $\begin{array}{l}\mathrm{PNI}(\leq 52.21 \text { vs. } \\
>52.21)\end{array}$ & $0.736(0.352-1.536)$ & 0.414 & $0.874(0.398-1.923)$ & 0.739 & $\begin{array}{l}1.111(0.421- \\
2.930)\end{array}$ & 0.832 & $\begin{array}{c}1.595(0.552- \\
4.612)\end{array}$ & 0.389 \\
\hline $\begin{array}{l}\text { SII }(\leq 324.55 \text { vs. } \\
>324.55)\end{array}$ & $6.416(3.438-11.980)$ & $<0.001$ & 8.549 (3.911-18.680) & $<0.001$ & $\begin{array}{c}2.951(1.350- \\
6.452)\end{array}$ & 0.007 & $\begin{array}{c}4.091(1.545- \\
10.833)\end{array}$ & 0.005 \\
\hline
\end{tabular}

OS, overall survival; RFS, recurrence-free survival; HR, hazard ratio; Cl, confidence interval; HBV, hepatitis B virus; AFP, alphafetoprotein; CTP, Child-Turcotte-Pugh; MELD, Model for End-Stage Liver Disease; ALB, albumin; ALT, alanine aminotransferase; AST, aspartic transaminase; $\gamma$-GT, $\gamma$-glutamyl transpeptidase; TBIL, total bilirubin; NLR, neutrophil lymphocyte ratio; SII, systemic immune inflammatory index; PLR, platelet lymphocyte ratio; PNI, prognostic nutritional index. 
Table 5 Baseline characteristics of patients with SII $\leq 324.55 \times 10^{9}$ versus SII $>324.55 \times 10^{9}$ in the training and validation cohorts

\begin{tabular}{|c|c|c|c|c|c|c|}
\hline Baseline characteristics & \multicolumn{3}{|c|}{ Training cohort } & \multicolumn{3}{|c|}{ Validation cohort } \\
\hline Age ( $\leq 50$ years) & 17 & 36 & 0.283 & 21 & 25 & 0.778 \\
\hline Gender (male) & 22 & 27 & 0.787 & 9 & 17 & 0.473 \\
\hline HBV (yes) & 80 & 127 & 0.704 & 52 & 70 & 1.000 \\
\hline Tumor number (single) & 68 & 79 & 0.014 & 42 & 31 & $<0.001$ \\
\hline Cirrhosis (yes) & 90 & 143 & 0.524 & 58 & 76 & 0.967 \\
\hline CTP (A) & 100 & 152 & 0.935 & 63 & 83 & 1.000 \\
\hline $\operatorname{MELD}(\leq 4.81)$ & 52 & 84 & 0.077 & 28 & 40 & 0.830 \\
\hline 2 & 45 & 75 & NA & 33 & 41 & NA \\
\hline 3 & 2 & 3 & NA & 0 & 1 & NA \\
\hline $\operatorname{AFP}(\leq 20$ ng/mL) & 78 & 70 & $<0.001$ & 58 & 25 & $<0.001$ \\
\hline $\operatorname{ALT}(\leq 40 \cup / L)$ & 76 & 115 & 0.966 & 50 & 63 & 0.672 \\
\hline AST ( $\leq 40$ U/L) & 82 & 133 & 0.418 & 56 & 67 & 0.209 \\
\hline$\gamma-\mathrm{GT}(\leq 60 \mathrm{U} / \mathrm{L})$ & 73 & 94 & 0.076 & 40 & 53 & 1.000 \\
\hline TBIL $(\leq 17.1 \mu \mathrm{mol} / \mathrm{L})$ & 63 & 96 & 1.000 & 60 & 77 & 0.603 \\
\hline ALB ( $\leq 43.2 \mathrm{~g} / \mathrm{L})$ & 84 & 128 & 1.000 & 48 & 67 & 0.751 \\
\hline
\end{tabular}

HBV, hepatitis B virus; AFP, alpha-fetoprotein; CTP, Child-Turcotte-Pugh; MELD, Model for End-Stage Liver Disease; ALB, albumin; ALT, alanine aminotransferase; AST, aspartic transaminase; $\gamma$-GT, $\gamma$-glutamyl transpeptidase; TBIL, total bilirubin.

1-, 2-, 3-, and 5-year RFS was 0.80 (95\% CI: 0.74-0.86), and the t-AUC for the nomograms predicting 1-, 2-, 3-, and 5-year RFS was $0.78,0.80,0.82$ and 0.83 , respectively (Figure $4 B$ ). In the validation cohort, the C-index for the likelihood of 1-, 2-, 3-, and 5-year RFS was 0.82 (95\% CI: 0.74-0.89), and the t-AUC for the nomograms predicting $1-, 2-, 3-$, and 5-year RFS was $0.82,0.83,0.75$, and 0.82 , respectively (Figure $4 C$ ). The results showed that the comprehensive nomogram was superior to the other clinical indexes in predicting prognosis in patients with HCC.

\section{Discussion}

In the current study, we found that SII, an inflammationrelated index was an independent predictor of long-term survival for early-stage HCC patients in two independent cohorts. This study is the first of its kind to demonstrate the relationship between SII and oncological outcomes in HCC patients treated with RFA. Previous studies proved the prognostic significance of SII for HCC patients undergoing chemoembolization (TACE), surgical resection, liver transplantation, and sorafenib treatment $(23,24,29)$. This study also provides a new SII-based scoring system with which to effectively evaluate the prognosis of HCC patients and select appropriate patients for RFA.

Cancer-related immune inflammation is now considered to be a key factor in the initiation, progression, and metastasis of HCC $(30,31)$. Our results showed that patients with high preoperative SII had shorter RFS and OS. The SII calculation includes peripheral blood platelet count, lymphocyte count, and neutrophil count. The predictive value of preoperative SII in HCC patients 

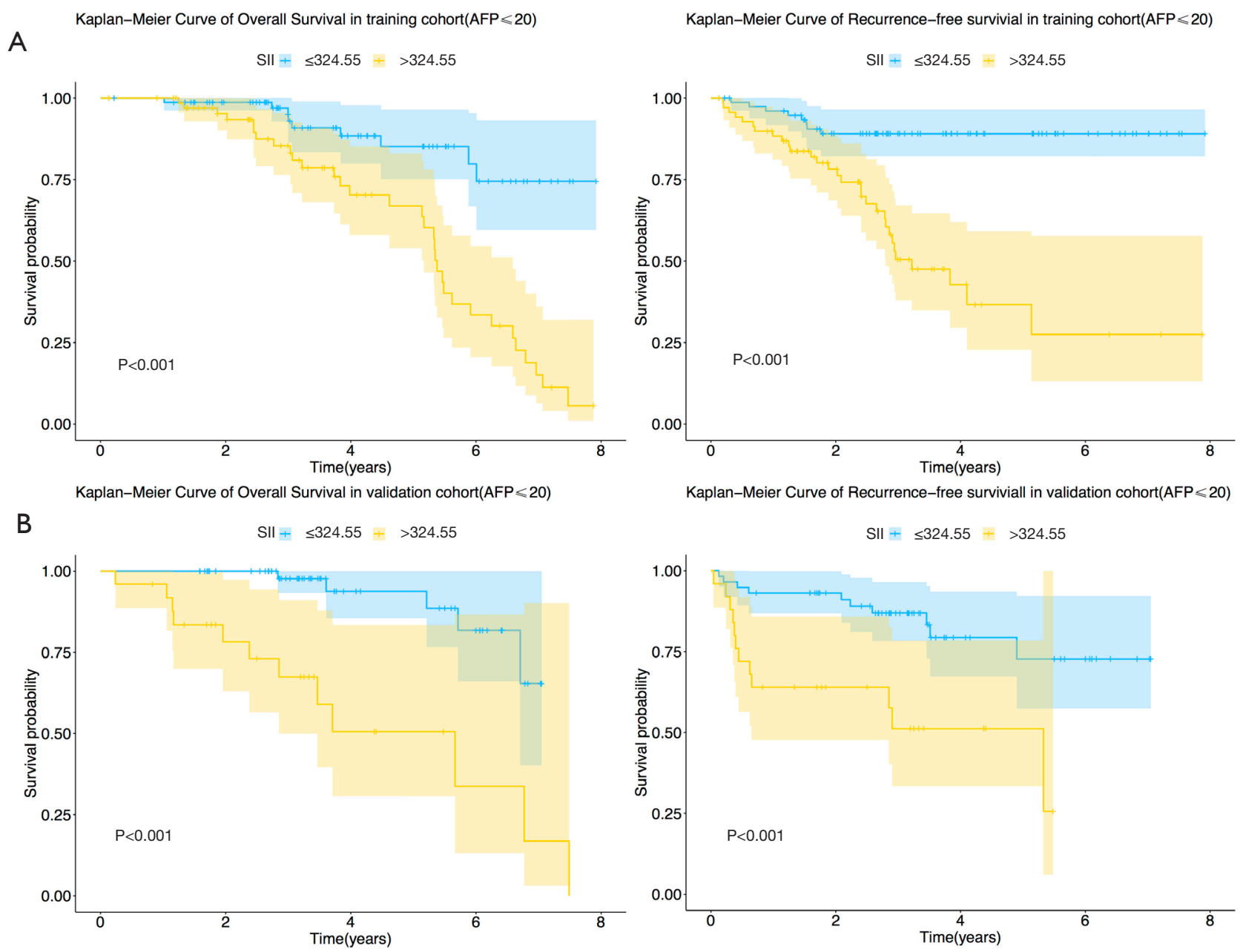

Figure 2 Prognostic significance of the SII in HCC patients with normal AFP levels (AFP <20 ng/mL). Kaplan-Meier analysis of RFS and OS based on the SII in the training (A) and validation (B) cohorts. SII, systemic immune-inflammation index; HCC, hepatocellular carcinoma; AFP, alpha-fetoprotein; OS, overall survival; RFS, recurrence-free survival.

may be elucidated by the interaction between these three types of inflammatory cells and tumor cells. Many studies have shown that neutrophils can promote the infiltration, reproduction, and metastasis of tumor tissue and aid cancer cells in escaping immune surveillance $(32,33)$. Platelets can interact with cancer cells through different mechanisms to promote tumor cell survival and metastasis $(34,35)$. On the other hand, lymphocytes release cytokines and induce cell death to inhibit tumor progression. Hence, low lymphocyte count impairs the host's immune response and accelerates tumor progression (36,37). Furthermore, this study showed that high SII was associated with early recurrence, multiple tumors, larger tumor size, and high levels of AFP, indicating more aggressive oncological characteristics and a poor prognosis.

SII can be regarded as a comprehensive index reflecting inflammation and activation of the immune pathway. Many studies have confirmed the prognostic value of PLR, and NLR in surgically resected HCC patients $(38-40)$. The results of this study indicated that the prediction accuracy of SII was superior to that of other inflammatory indexes, such as PLR and NLR. An elevated SII signified relative neutrophilia, thrombocytosis, and lymphocytopenia, suggesting an increased inflammatory state but decreased immune response to the tumor. HCC is a common inflammation-induced cancer in patients 


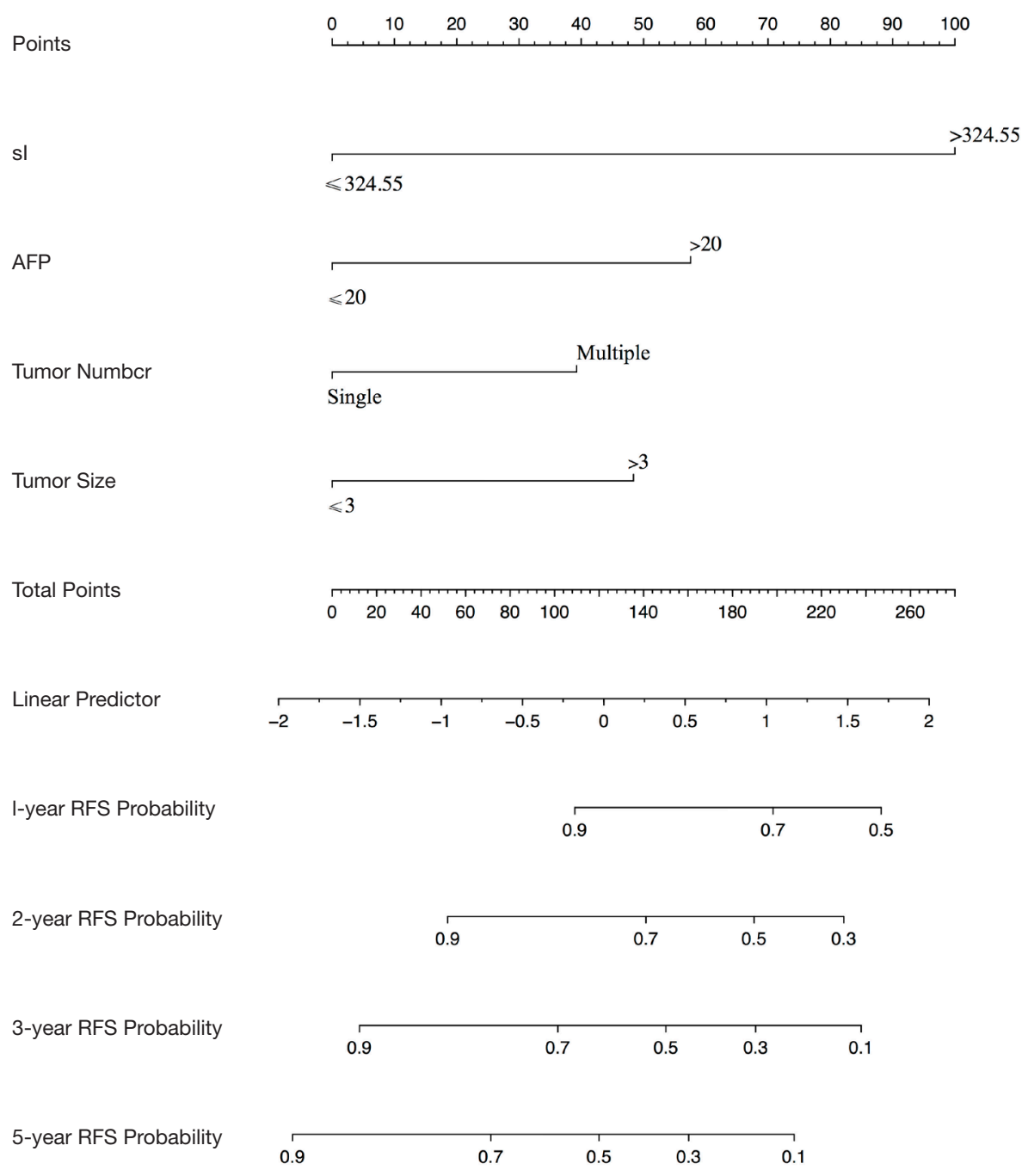

Figure 3 Nomogram for predicting 1-, 2-, 3-, and 5-year RFS probability based on the SII. SII, systemic immune-inflammation index; RFS, recurrence-free survival.

with HBV infection (41). Understanding the value of SII in the occurrence and development of HCC will help us in understanding the relationship between HCC and the immune inflammatory response. We believe that HCC patients with increased SII may benefit from targeted drug therapy to regulate immunity and inflammation, such as thymosin and aspirin $(42,43)$.

AFP is widely used to diagnose and prognosticate HCC. However, about $30 \%$ of patients with early-stage HCC have normal AFP levels $(44,45)$. There is no ideal biomarker to predict the survival outcomes of early-stage HCC patients with normal AFP levels. In this study, the results of subgroup analysis showed that high SII was associated with poor OS and RFS, even in early-stage HCC patients with normal AFP levels. These findings suggest that SII may be a useful marker for predicting survival in these patients. Moreover, it will help clinicians to identify highrisk patients who may benefit from adjuvant therapy after RFA to prevent recurrence.

Many comprehensive nomograms have been widely used as a visualization tool to predict survival and recurrence in patients with various types of tumors, including HCC (46). In this study, we established a comprehensive nomogram based on SII score and other commonly used prognostic factors to predict oncological outcomes in patients with early-stage HCC undergoing ablation. Notably, the follow- 

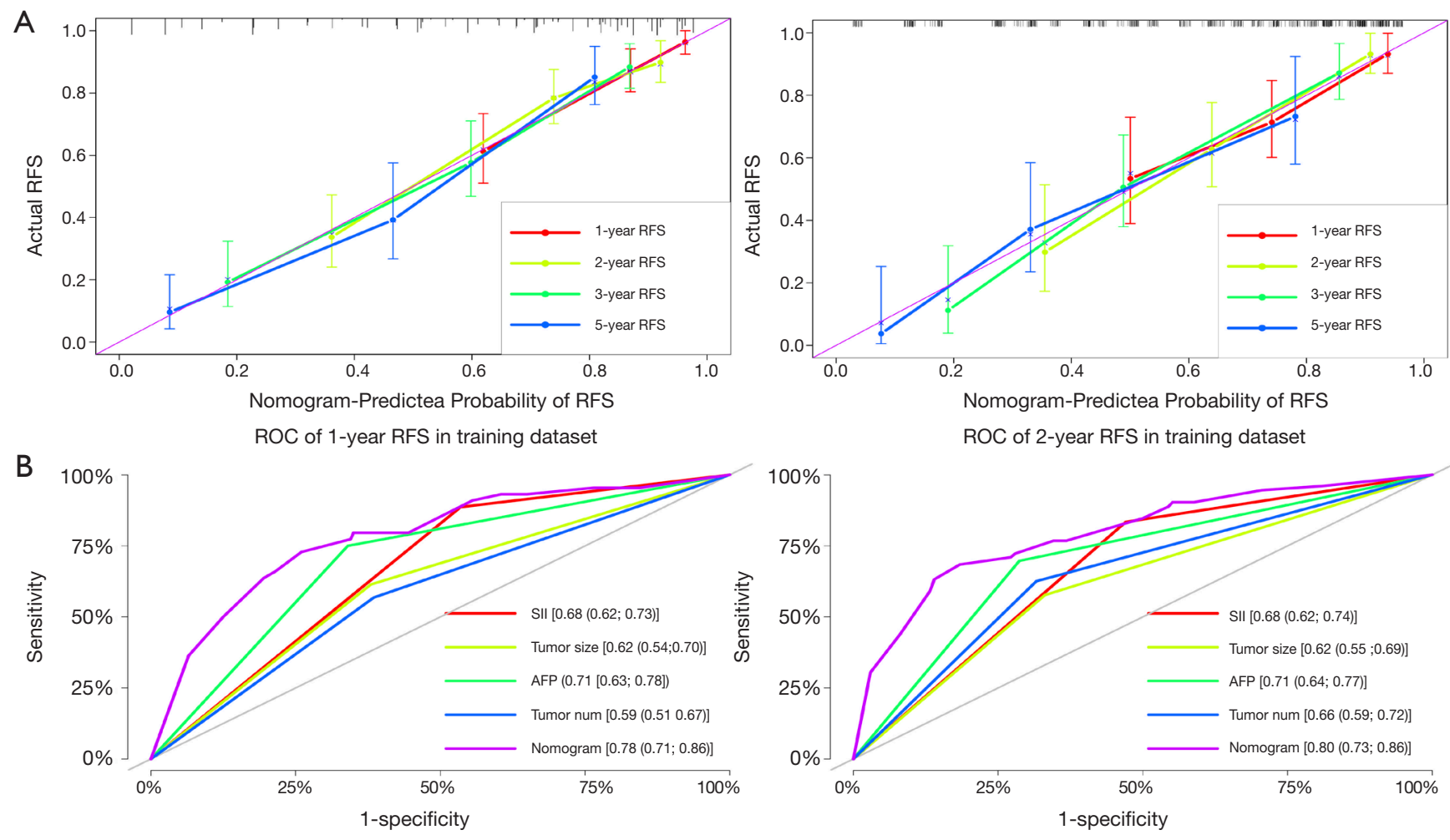

ROC of 3-year RFS in training dataset

$\mathrm{ROC}$ of 5-year RFS in training dataset
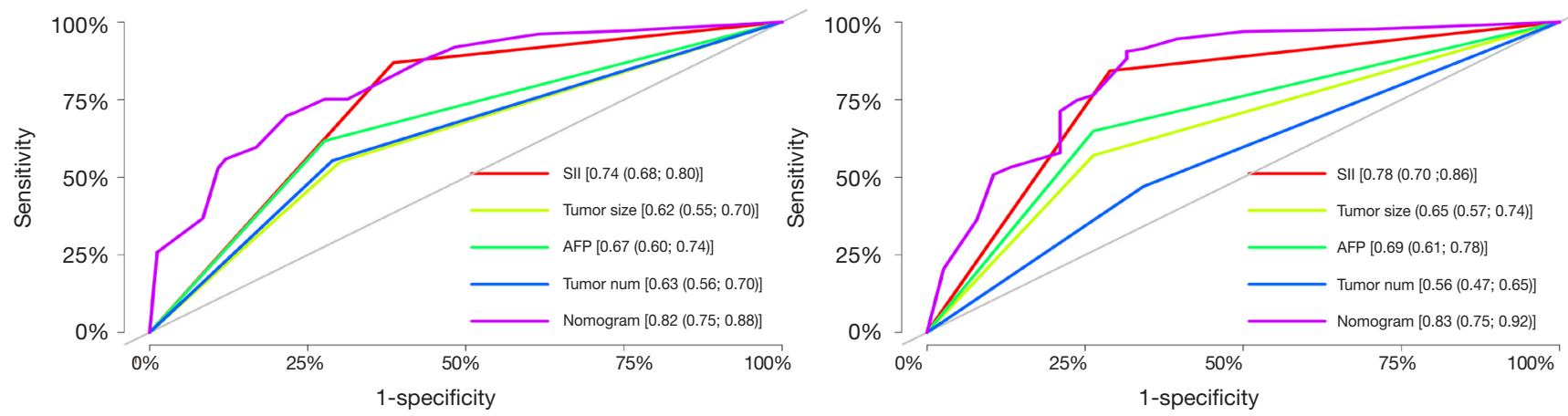

(c) Journal of Gastrointestinal Oncology. All rights reserved. 
ROC of 1-year RFS in validation dataset

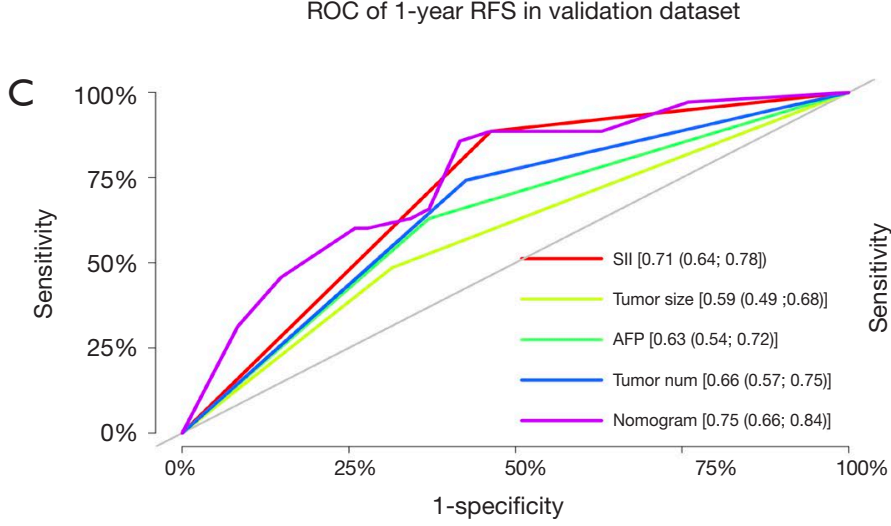

ROC of 3-year RFS in validation dataset

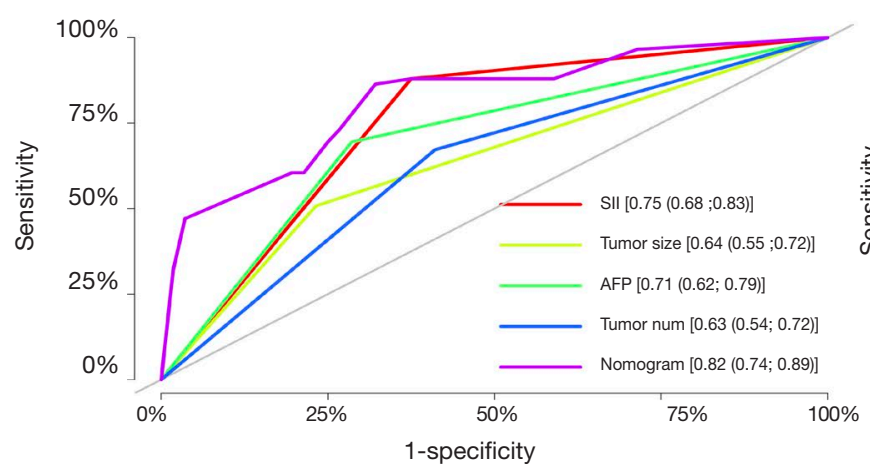

ROC of 2-year RFS in validation dataset

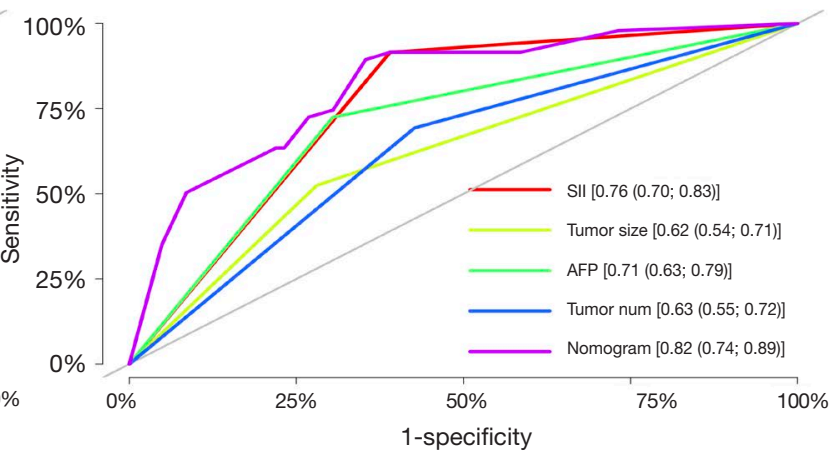

ROC of 5-year RFS in validation dataset

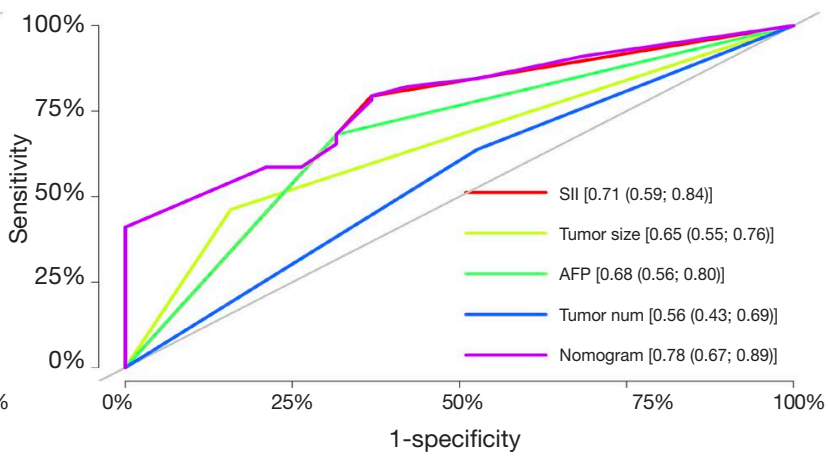

Figure 4 Calibration curves for predicting 1-, 2-, 3- and 5-year RFS in the training and validation cohorts (A). Time-dependent ROC curves by nomogram and AFP/tumor number/size for 1-, 2-, 3-, and 5-year RFS in the training (B) and validation cohorts (C). RFS, recurrence-free survival; ROC, receiver operating characteristic; AFP, alpha-fetoprotein.

up treatment of HCC patients after RFA was inconsistent, which significantly affected OS. Therefore, predictive nomograms for RFS only were constructed. The five prognostic factors included in the nomogram for RFS were tumor size, tumor number, AFP level, and SII score. The Barcelona Clinic Liver Cancer (BCLC) system is a scoring system commonly used to evaluate prognosis in HCC patients (47). However, it has been reported that patients with BCLC of the same stage may show significantly different clinical symptoms, leading to different tumor outcomes (48). Therefore, better prognostic models are needed to improve the predictive performance of the BCLC system. Although the BCLC system includes tumor burden, clinical status, liver function parameters, and other factors, it does not include inflammatory indices. In view of the key role of immune inflammatory factors in the occurrence and progression of HCC, we established a comprehensive nomogram including inflammatory index and other conventional factors that may provide increased prognostic value for HCC patients.

The present study has some limitations. First, the study was retrospective in nature, with limited sample size and inherent selection bias. Future multicenter, prospective studies are necessary to confirm the findings of this study. Second, the choice of patients, HCC treatment protocol, and follow-up strategies varied from center to center. Third, there was some heterogeneity in the treatment of HCC recurrence after RFA at the study centers, which may have directly affected the long-term prognosis of the patients. So, in this study, we constructed a nomogram for the prediction of RFS only in HCC patients. Lastly, most of the study patients had HBV-related HCC. Hence, the results cannot be extrapolated to other populations, such as Western countries where alcohol and non-alcoholic steatohepatitis are the most common causes of HCC (11).

In summary, the current study is the first to explore the 
prognostic significance of SII score in early-stage HCC patients after RFA. The preliminary results of this dualcenter study confirmed that SII, a novel comprehensive index, was an independent predictor for RFS and OS among early-stage HCC patients after RFA. Moreover, the SII was superior to other conventional indexes. The comprehensive SII-based nomogram presented above may help clinicians to identify high-risk patients and to formulate individualized treatment plans. The SII is a potential biomarker for the prediction of prognosis in HCC due to its comprehensiveness, simplicity, and low cost in clinical practice.

\section{Acknowledgments}

Funding: This work was supported by National Natural Science Foundation of China (30970839 \& 31170957); and Research Project of Shanxi Province Health Commission (2017045).

\section{Footnote}

Reporting Checklist: The authors have completed the STROBE reporting checklist. Available at: http://dx.doi. org/10.21037/jgo-20-342

Data Sharing Statement: Available at: http://dx.doi. org/10.21037/jgo-20-342

Conflicts of Interest: All authors have completed the ICMJE uniform disclosure form (available at: http://dx.doi. org/10.21037/jgo-20-342). The authors have no conflicts of interest to declare.

Ethical Statement: The authors are accountable for all aspects of the work in ensuring that questions related to the accuracy or integrity of any part of the work are appropriately investigated and resolved. The study was conducted in accordance with the Declaration of Helsinki (as revised in 2013). The study was approved by the Institutional Review Boards of the National Cancer Center (NCC2019KZ-010). Written informed consent for treatment was obtained from each patient. The need for written informed consent to publish the data was waived by the Institutional Review Boards, since the personal details of these patients were kept confidential.

Open Access Statement: This is an Open Access article distributed in accordance with the Creative Commons Attribution-NonCommercial-NoDerivs 4.0 International License (CC BY-NC-ND 4.0), which permits the noncommercial replication and distribution of the article with the strict proviso that no changes or edits are made and the original work is properly cited (including links to both the formal publication through the relevant DOI and the license). See: https://creativecommons.org/licenses/by-nc-nd/4.0/.

\section{References}

1. Bray F, Ferlay J, Soerjomataram I, et al. Global cancer statistics 2018: GLOBOCAN estimates of incidence and mortality worldwide for 36 cancers in 185 countries. CA Cancer J Clin 2018;68:394-424.

2. McGlynn KA, Petrick JL, El-Serag HB. Epidemiology of Hepatocellular Carcinoma. Hepatology 2021;73 Suppl 1:4-13.

3. Chen $\mathrm{W}$, Zheng R, Baade PD, et al. Cancer statistics in China, 2015. CA Cancer J Clin 2016;66:115-32.

4. Kang D, Shim S, Cho J, et al. Systematic Review of Studies Assessing the Health-Related Quality of Life of Hepatocellular Carcinoma Patients from 2009 to 2018. Korean J Radiol 2020;21:633-46.

5. Guidelines for diagnosis and treatment of primary liver cancer in China (2019 edition). Zhonghua Gan Zang Bing Za Zhi 2020;28:112-28.

6. Rajyaguru DJ, Borgert AJ, Smith AL, et al. Radiofrequency Ablation Versus Stereotactic Body Radiotherapy for Localized Hepatocellular Carcinoma in Nonsurgically Managed Patients: Analysis of the National Cancer Database. J Clin Oncol 2018;36:600-8.

7. Xia Y, Li J, Liu G, et al. Long-term Effects of Repeat Hepatectomy vs Percutaneous Radiofrequency Ablation Among Patients With Recurrent Hepatocellular Carcinoma: A Randomized Clinical Trial. JAMA Oncol 2020;6:255-63.

8. Raoul JL, Edeline J. Systemic treatment of hepatocellular carcinoma: standard of care in China and elsewhere. Lancet Oncol 2020;21:479-81.

9. Gunasekaran G, Bekki Y, Lourdusamy V, et al. Surgical Treatments of Hepatobiliary Cancers. Hepatology 2021;73 Suppl 1:128-136.

10. Vibert E, Schwartz M, Olthoff KM. Advances in resection and transplantation for hepatocellular carcinoma. J Hepatol 2020;72:262-76.

11. El-Serag HB, Rudolph KL. Hepatocellular carcinoma: epidemiology and molecular carcinogenesis. 
Gastroenterology 2007;132:2557-76.

12. Xu XL, Liu XD, Liang M, et al. Radiofrequency Ablation versus Hepatic Resection for Small Hepatocellular Carcinoma: Systematic Review of Randomized Controlled Trials with Meta-Analysis and Trial Sequential Analysis. Radiology 2018;287:461-72.

13. Wang JH, Wang CC, Hung CH, et al. Survival comparison between surgical resection and radiofrequency ablation for patients in BCLC very early/early stage hepatocellular carcinoma. J Hepatol 2012;56:412-8.

14. Feng J, Qi X, Guo X, et al. Early-Stage Hepatocellular Carcinoma: Radiofrequency Ablation or Resection? Am J Gastroenterol 2019;114:359.

15. Mohkam K, Dumont PN, Manichon AF, et al. No-touch multibipolar radiofrequency ablation vs. surgical resection for solitary hepatocellular carcinoma ranging from 2 to 5 cm. J Hepatol 2018;68:1172-80.

16. Kobayashi N, Usui S, Kikuchi S, et al. Preoperative lymphocyte count is an independent prognostic factor in node-negative non-small cell lung cancer. Lung Cancer 2012;75:223-7.

17. Souza Cunha M, Wiegert EVM, Calixto-Lima L, et al. Relationship of nutritional status and inflammation with survival in patients with advanced cancer in palliative care. Nutrition 2018;51-52:98-103.

18. Singh R, Mishra MK, Aggarwal H. Inflammation, Immunity, and Cancer. Mediators Inflamm 2017;2017:6027305.

19. Hanahan D, Weinberg RA. Hallmarks of cancer: the next generation. Cell 2011;144:646-74.

20. Shi H, Jiang Y, Cao H, et al. Nomogram Based on Systemic Immune-Inflammation Index to Predict Overall Survival in Gastric Cancer Patients. Dis Markers 2018;2018:1787424.

21. Chen JH, Zhai ET, Yuan YJ, et al. Systemic immuneinflammation index for predicting prognosis of colorectal cancer. World J Gastroenterol 2017;23:6261-72.

22. Yang R, Chang Q, Meng X, et al. Prognostic value of Systemic immune-inflammation index in cancer: A metaanalysis. J Cancer 2018;9:3295-302.

23. Hu B, Yang XR, Xu Y, et al. Systemic immuneinflammation index predicts prognosis of patients after curative resection for hepatocellular carcinoma. Clin Cancer Res 2014;20:6212-22.

24. Fu H, Zheng J, Cai J, et al. Systemic ImmuneInflammation Index (SII) is Useful to Predict Survival Outcomes in Patients After Liver Transplantation for Hepatocellular Carcinoma within Hangzhou Criteria. Cell
Physiol Biochem 2018;47:293-301.

25. Yang Z, Zhang J, Lu Y, et al. Aspartate aminotransferaselymphocyte ratio index and systemic immuneinflammation index predict overall survival in $\mathrm{HBV}$-related hepatocellular carcinoma patients after transcatheter arterial chemoembolization. Oncotarget 2015;6:43090-8.

26. Wang BL, Tian L, Gao XH, et al. Dynamic change of the systemic immune inflammation index predicts the prognosis of patients with hepatocellular carcinoma after curative resection. Clin Chem Lab Med 2016;54:1963-9.

27. Pang Q, Zhou L, Qu K, et al. Validation of inflammationbased prognostic models in patients with hepatitis B-associated hepatocellular carcinoma: a retrospective observational study. Eur J Gastroenterol Hepatol 2018;30:60-70.

28. EASL-EORTC clinical practice guidelines: management of hepatocellular carcinoma. Eur J Cancer 2012;48:599-641.

29. Casadei Gardini A, Scarpi E, Faloppi L, et al. Immune inflammation indicators and implication for immune modulation strategies in advanced hepatocellular carcinoma patients receiving sorafenib. Oncotarget 2016;7:67142-9.

30. Kanda T, Goto T, Hirotsu Y, et al. Molecular Mechanisms Driving Progression of Liver Cirrhosis towards Hepatocellular Carcinoma in Chronic Hepatitis B and C Infections: A Review. Int J Mol Sci 2019;20:1358.

31. Alison MR, Nicholson LJ, Lin WR. Chronic inflammation and hepatocellular carcinoma. Recent Results Cancer Res 2011;185:135-48.

32. Diakos CI, Charles KA, McMillan DC, et al. Cancerrelated inflammation and treatment effectiveness. Lancet Oncol 2014;15:e493-503.

33. Mantovani A, Allavena P, Sica A, et al. Cancer-related inflammation. Nature 2008;454:436-44.

34. Labelle M, Begum S, Hynes RO. Direct signaling between platelets and cancer cells induces an epithelialmesenchymal-like transition and promotes metastasis. Cancer Cell 2011;20:576-90.

35. Schumacher D, Strilic B, Sivaraj KK, et al. Plateletderived nucleotides promote tumor-cell transendothelial migration and metastasis via P2Y2 receptor. Cancer Cell 2013;24:130-7.

36. Ferrone C, Dranoff G. Dual roles for immunity in gastrointestinal cancers. J Clin Oncol 2010;28:4045-51.

37. Chew V, Chen J, Lee D, et al. Chemokine-driven lymphocyte infiltration: an early intratumoural event determining long-term survival in resectable hepatocellular carcinoma. Gut 2012;61:427-38. 
38. Kinoshita A, Onoda H, Imai N, et al. Comparison of the prognostic value of inflammation-based prognostic scores in patients with hepatocellular carcinoma. Br J Cancer 2012;107:988-93.

39. Mano Y, Shirabe K, Yamashita Y, et al. Preoperative neutrophil-to-lymphocyte ratio is a predictor of survival after hepatectomy for hepatocellular carcinoma: a retrospective analysis. Ann Surg 2013;258:301-5.

40. Wang D, Hu X, Xiao L, et al. Prognostic Nutritional Index and Systemic Immune-Inflammation Index Predict the Prognosis of Patients with HCC. J Gastrointest Surg 2021;25:421-7.

41. Bishayee A. The role of inflammation and liver cancer. Adv Exp Med Biol 2014;816:401-35.

42. Sahasrabuddhe VV, Gunja MZ, Graubard BI, et al. Nonsteroidal anti-inflammatory drug use, chronic liver disease, and hepatocellular carcinoma. J Natl Cancer Inst 2012;104:1808-14.

43. Sjogren MH. Thymalfasin: an immune system enhancer for the treatment of liver disease. J Gastroenterol Hepatol
2004;19 Suppl 6:S69-72.

44. Luo P, Wu S, Yu Y, et al. Current Status and Perspective Biomarkers in AFP Negative HCC: Towards Screening for and Diagnosing Hepatocellular Carcinoma at an Earlier Stage. Pathol Oncol Res 2020;26:599-603.

45. Shu H, Li W, Shang S, et al. Diagnosis of AFP-negative early-stage hepatocellular carcinoma using Fuc-PON1. Discov Med 2017;23:163-8.

46. Chen SH, Wan QS, Zhou D, et al. A Simple-toUse Nomogram for Predicting the Survival of Early Hepatocellular Carcinoma Patients. Front Oncol 2019;9:584.

47. Llovet JM, Brú C, Bruix J. Prognosis of hepatocellular carcinoma: the BCLC staging classification. Semin Liver Dis 1999;19:329-38.

48. Yoo JJ, Chung GE, Lee JH, et al. Sub-classification of Advanced-Stage Hepatocellular Carcinoma: A Cohort Study Including 612 Patients Treated with Sorafenib. Cancer Res Treat 2018;50:366-73.

Cite this article as: Xin Y, Yang Y, Liu N, Chen Y, Wang Y, Zhang X, Li X, Zhou X. Prognostic significance of systemic immune-inflammation index-based nomogram for early stage hepatocellular carcinoma after radiofrequency ablation. J Gastrointest Oncol 2021;12(2):735-750. doi: 10.21037/jgo-20-342 